\title{
SPIN QUANTUM PLASMAS - NEW ASPECTS OF COLLECTIVE DYNAMICS
}

\author{
M. MARKLUND* and G. BRODIN \\ Department of Physics, Umeå University, SE-901 87 Umeå, Sweden \\ *E-mail: mattias.marklund@physics.umu.se
}

\begin{abstract}
Quantum plasmas is a rapidly expanding field of research, with applications ranging from nanoelectronics, nanoscale devices and ultracold plasmas, to inertial confinement fusion and astrophysics. Here we give a short systematic overview of quantum plasmas. In particular, we analyze the collective effects due to spin using fluid models. The introduction of an intrinsic magnetization due to the plasma electron (or positron) spin properties in the magnetohydrodynamic limit is discussed. Finally, a discussion of the theory and examples of applications is given.
\end{abstract}

\section{Introduction}

The field of quantum plasmas is a rapidly growing field of research. From the non-relativistic domain, with its basic description in terms of the Schrödinger equation, to the strongly relativistic regime, with its natural connection to quantum field theory, quantum plasma physics provides promises of highly interesting and important application, fundamental connections between different areas of science, as well as difficult challenges from a computational perspective. The necessity to thoroughly understand such plasmas motivates a reductive principle of research, for which we successively build more complex models based on previous results. The simplest lower order effect due to relativistic quantum mechanics is the introduction of spin, and as such thus provides a first step towards a partial description of relativistic quantum plasmas.

Already in the 1960's, Pines studied the excitation spectrum of quantum plasmas, ${ }^{1,2}$ for which we have a high density and a low temperature as compared to normal plasmas. In such systems, the finite width of the electron wave function makes quantum tunnelling effects crucial, leading to an altered dispersion relation. Since the pioneering work by Pines, a number or theoretical studies of quantum statistical properties of plasmas has been 
done (see, e.g., Ref. 3 and references therein). For example, Bezzerides \& DuBois presented a kinetic theory for the quantum electrodynamical properties of nonthermal plasmas, ${ }^{4}$ while Hakim \& Heyvaerts presented a covariant Wigner function approach for relativistic quantum plasmas. ${ }^{5}$ Recently there has been an increased interest in the properties of quantum plasmas. ${ }^{6-25}$ The studies has been motivated by the development in nanostructured materials ${ }^{26}$ and quantum wells, ${ }^{27}$ the discovery of ultracold plasmas $^{28}$ (see Ref. 29 for an experimental demonstration of quantum plasma oscillations in Rydberg systems), astrophysical applications, ${ }^{30}$ or a general theoretical interest. Moreover, it has recently been experimentally shown that quantum dispersive effects are important in inertial confinement plasmas. ${ }^{31}$ The list of quantum mechanical effects that can be included in a fluid picture includes the dispersive particle properties accounted for by the Bohm potential, ${ }^{6-18}$ the zero temperature Fermi pressure, ${ }^{6-10}$ spin properties $^{19-21}$ as well as certain quantum electrodynamical effects. ${ }^{32-35}$ Within such descriptions, ${ }^{6-10,19,20,33-35}$ quantum and classical collective effects can be described within a unified picture.

\section{The microscopic equations: Schrödinger and Pauli dynamics}

\subsection{The Schrödinger description}

The basic equation of nonrelativistic quantum mechanics is the Schrödinger equation. The dynamics of an electron, represented by its wave function $\psi$, in an external electromagnetic potential $\phi$ is governed by

$$
i \hbar \frac{\partial \psi}{\partial t}+\frac{\hbar}{2 m_{e}} \nabla^{2} \psi+e \phi \psi=0
$$

where $\hbar$ is Planck's constant, $m_{e}$ is the electron mass, and $e$ is the magnitude of the electron charge. This complex equation may be written as two real equations, writing $\psi=\sqrt{n} \exp i S / \hbar$, where $n$ is the amplitude and $S$ the phase of the wave function, respectively. ${ }^{36}$ Such a decomposition was presented by de Broglie and Bohm in order to understand the dynamics of the electron wave packet in terms of classical variables. Using this decomposition in Eq. (1), we obtain

$$
\frac{\partial n}{\partial t}+\nabla \cdot(n \mathbf{v})=0
$$

and

$$
m_{e} \frac{d \mathbf{v}}{d t}=e \nabla \phi+\frac{\hbar^{2}}{2 m_{e}} \nabla\left(\frac{\nabla^{2} \sqrt{n}}{\sqrt{n}}\right)
$$


where the velocity is defined by $\mathbf{v}=\nabla S / m_{e}$. The last term of Eq. (3) is the gradient of the Bohm-de Broglie potential, and is due to the effect of wave function spreading, giving rise to a dispersive-like term. We also note the striking resemblance of Eqs. (2) and (3) to the classical fluid equations.

\subsection{The Pauli description}

In relativistic quantum mechanics, the spin of the electron (and positron) is rigorously introduced through the Dirac Hamiltonian

$$
H=c \alpha \cdot(\mathbf{p}+e \mathbf{A})-e \phi+\beta m_{e} c^{2},
$$

where $\alpha=\left(\alpha_{1}, \alpha_{2}, \alpha_{3}\right), e$ is the magnitude of the electron charge, $c$ is the speed of light, $\mathbf{A}$ is the vector potential, $\phi$ is the electrostatic potential, and the relevant matrices are given by

$$
\alpha=\left(\begin{array}{cc}
0 & \sigma \\
\sigma & 0
\end{array}\right), \quad \beta=\left(\begin{array}{cc}
\mathrm{I} & 0 \\
0 & -\mathrm{I}
\end{array}\right) .
$$

Here I is the unit $2 \times 2$ matrix and $\sigma=\left(\sigma_{1}, \sigma_{2}, \sigma_{3}\right)$, where we have the Pauli spin matrices

$$
\sigma_{1}=\left(\begin{array}{ll}
0 & 1 \\
1 & 0
\end{array}\right), \sigma_{2}=\left(\begin{array}{cc}
0 & -i \\
i & 0
\end{array}\right), \text { and } \sigma_{3}=\left(\begin{array}{cc}
1 & 0 \\
0 & -1
\end{array}\right) .
$$

From the Hamiltonian (4), a nonrelativistic counterpart may be obtained, taking the form

$$
H=\frac{1}{2 m_{e}}(\mathbf{p}+e \mathbf{A})^{2}+\frac{e \hbar}{2 m_{e}} \mathbf{B} \cdot \sigma-e \phi .
$$

Thus, the electron possesses a magnetic moment $\mathbf{m}=-\mu_{B}\langle\psi|\sigma| \psi\rangle /\langle\psi \mid \psi\rangle$, where $\mu_{B}=e \hbar / 2 m_{e}$ is the Bohr magneton, giving a contribution $-\mathbf{B} \cdot \mathbf{m}$ to the energy. The latter shows the paramagnetic property of the electron, where the spin vector is anti-parallel to the magnetic field in order to minimize the energy of the magnetized system. According to (7) and the relation $d F / d t=\partial F / \partial t+(1 / i \hbar)[F, H]$, where $F$ is some operator and [,] is the Poisson bracket, we have the following evolution equations for the position and momentum in the Heisenberg picture ${ }^{36-38}$

$$
\begin{gathered}
\mathbf{v} \equiv \frac{d \mathbf{x}}{d t}=\frac{1}{m_{e}}(\mathbf{p}+e \mathbf{A}), \\
m_{e} \frac{d \mathbf{v}}{d t}=-e(\mathbf{E}+\mathbf{v} \times \mathbf{B})-\frac{2}{\hbar} \mu_{B} \nabla(\mathbf{B} \cdot \mathbf{s}),
\end{gathered}
$$


while the spin evolution is given by

$$
\frac{d \mathbf{s}}{d t}=\frac{2}{\hbar} \mu_{B} \mathbf{B} \times \mathbf{s}
$$

where the spin operator is given by

$$
\mathbf{s}=\frac{\hbar}{2} \sigma .
$$

The above equations thus gives the quantum operator equivalents of the equations of motion for a classical particle, including the evolution of the spin in a magnetic field.

The non-relativistic evolution of spin $\frac{1}{2}$ particles, as described by the two-component spinor $\Psi_{(\alpha)}$, is given by the Pauli equation (see, e.g., 36)

$$
i \hbar \frac{\partial \psi}{\partial t}+\left[\frac{\hbar^{2}}{2 m_{e}}\left(\nabla+\frac{i e}{\hbar} \mathbf{A}\right)^{2}-\mu_{B} \mathbf{B} \cdot \sigma+e \phi\right] \psi=0
$$

where $\mathbf{A}$ is the vector potential, $\mu_{B}=e \hbar / 2 m_{e}$ is the Bohr magneton, and $\sigma=\left(\sigma_{1}, \sigma_{2}, \sigma_{3}\right)$ is the Pauli spin vector.

Now, in the same way as in the Schrödinger case, we may decompose the electron wave function $\psi$ into its amplitude and phase. However, as the electron has spin, the wave function is now represented by a 2 -spinor instead of a c-number. Thus, we may use $\psi=\sqrt{n} \exp (i S / \hbar) \varphi$, where $\varphi$, normalized such that $\varphi^{\dagger} \varphi=1$, now gives the spin part of the wave function. Multiplying the Pauli equation (12) by $\psi^{\dagger}$, inserting the above wave function decomposition and taking the gradient of the resulting phase evolution equation, we obtain the conservation equations

$$
\frac{\partial n}{\partial t}+\nabla \cdot(n \mathbf{v})=0
$$

and

$$
\begin{aligned}
m_{e} \frac{d \mathbf{v}}{d t}= & -e(\mathbf{E}+\mathbf{v} \times \mathbf{B})+\frac{\hbar^{2}}{2 m_{e}} \nabla\left(\frac{\nabla^{2} \sqrt{n}}{\sqrt{n}}\right) \\
& -\frac{2 \mu_{B}}{\hbar}(\nabla \otimes \mathbf{B}) \cdot \mathbf{s}-\frac{1}{m_{e} n} \nabla \cdot(n \boldsymbol{\Sigma})
\end{aligned}
$$

respectively. The spin contribution to Eq. (14) is consistent with the results of Ref. 39. Here the velocity is defined by

$$
\mathbf{v}=\frac{1}{m_{e}}\left(\nabla S-i \hbar \varphi^{\dagger} \nabla \varphi\right)+\frac{e \mathbf{A}}{m_{e} c},
$$

the spin density vector is

$$
\mathbf{s}=\frac{\hbar}{2} \varphi^{\dagger} \sigma \varphi
$$


which is normalized according to

$$
|\mathbf{s}|=\hbar / 2
$$

and we have defined the symmetric gradient spin tensor

$$
\boldsymbol{\Sigma}=\left(\nabla s_{a}\right) \otimes\left(\nabla s^{a}\right)
$$

Moreover, contracting Eq. (12) by $\psi^{\dagger} \sigma$, we obtain the spin evolution equation

$$
\frac{d \mathbf{s}}{d t}=\left\{\frac{2 \mu_{B}}{\hbar} \mathbf{B}-\frac{1}{m_{e} n}\left[\partial_{a}\left(n \partial^{a} \mathbf{s}\right)\right]\right\} \times \mathbf{s} .
$$

We note that the last equation allows for the introduction of an effective magnetic field $\mathbf{B}_{\text {eff }} \equiv\left(2 \mu_{B} / \hbar\right) \mathbf{B}-\left(m_{e} n\right)^{-1}\left[\partial_{a}\left(n \partial^{a} \mathbf{s}\right)\right]$. However, this will not pursued further here (for a discussion, see Ref. 36).

Comparing the effects due to spin from the Pauli dynamics with the Schrödinger theory, we see a significant increase in the complexity of the fluid like equations due the presence of spin. The fact that the spin couples linearly to the magnetic field makes the dynamical aspects of such Pauli systems very rich. Moreover, when going over to the collective regime, the back reaction through Maxwell's equation can yield interesting new properties of such spin plasmas. In fact, the introduction of an intrinsic magnetization can give rise to linear instability regimes, much like the Jeans instability (see Sec. 5.2.).

\section{Collective plasma dynamics}

As pointed out in the previous section, the route from single wavefunction dynamics to collective effects introduces a new complexity into the system. At the classical level, the ordinary pressure is such an effect. In the quantum case, a similar term, based on the thermal distribution of spins, will be introduced.

\subsection{Multistream model}

The multistream model of classical plasmas was successfully introduced by Dawson. ${ }^{40}$ Here we will focus on the electrostatic interaction between a multistream quantum plasma described within the Schrödiner model, a system first investigated in Ref. 7 (where also the stationary regime was probed). Thus, we have the governing equations (2) and (3) but for $N$ beams 
of electrons on a stationary ion background, i.e., Using this decomposition in Eq. (1), we obtain

$$
\frac{\partial n_{\alpha}}{\partial t}+\nabla \cdot\left(n_{\alpha} \mathbf{v}_{\alpha}\right)=0
$$

and

$$
m_{e} \frac{d \mathbf{v}_{\alpha}}{d t}=e \nabla \phi+\frac{\hbar^{2}}{2 m_{e}} \nabla\left(\frac{\nabla^{2} \sqrt{n_{\alpha}}}{\sqrt{n_{\alpha}}}\right),
$$

now coupled through the self-consistent electrostatic potential governed by

$$
\nabla^{2} \phi=\frac{e}{\epsilon_{0}} \sum_{\alpha=1}^{N}\left(n_{\alpha}-n_{0}\right)
$$

Here, $d / d t=\partial_{t}+\mathbf{v}_{\alpha} \cdot \nabla$ and $n_{0}$ is the density of the stationary ion background.

In the one-stream case $(\alpha=1)$, we have the equilibrium solution $\mathbf{v}=\mathbf{v}_{0}$ (a constant drift relative the stationary ion background) and the constant electron density $n=n_{0}$ (such that $\phi=0$ ). Perturbing this system a Fourier decomposing the perturbations, such that $\left.n=n_{0}+\delta n \exp [i(\mathbf{k} \cdot \mathbf{x}-\omega t)]\right)$, $\mathbf{v}=\mathbf{v}_{0}+\delta \mathbf{v} \exp [i(\mathbf{k} \cdot \mathbf{x}-\omega t)]$, and $\phi=\delta \phi \exp [i(\mathbf{k} \cdot \mathbf{x}-\omega t)]$, we obtain ${ }^{1,7}$

$$
\left(\omega-\mathbf{k} \cdot \mathbf{v}_{0}\right)^{2}=\omega_{p}^{2}+\frac{\hbar^{2} k^{4}}{4 m_{e}^{2}}
$$

where the last term is the Bohm-de Broglie correction to the dispersion relation. Here we have the electron plasma frequency $\omega_{p}=\left(e^{2} n_{0} / \epsilon_{0} m_{e}\right)^{1 / 2}$.

Similarly to the one-stream case, we obtain the dispersion relation ${ }^{7,41}$

$$
\begin{aligned}
1=\frac{\omega_{p 1}^{2}}{\left(\omega-\mathbf{v}_{01} \cdot \mathbf{k}\right)^{2}-\hbar^{2} k^{4} / 4 m_{e}^{2}} \\
\quad+\frac{\omega_{p 2}^{2}}{\left(\omega-\mathbf{v}_{02} \cdot \mathbf{k}\right)^{2}-\hbar^{2} k^{4} / 4 m_{e}^{2}},
\end{aligned}
$$

for two propagating electron beams (with velocities $\mathbf{v}_{01}$ and $\mathbf{v}_{02}$ ) with background densities $n_{01}$ and $n_{02}$. The quantum effect has a subtle influence on the stability of the perturbed plasma. For the case $n_{01}=n_{02}=n_{0} / 2$ and $\mathbf{v}_{01}=-\mathbf{v}_{02}=\mathbf{v}_{0}$, we have the instability condition

$$
\frac{4}{K^{2}}\left(1-\frac{1}{K^{2}}\right)<H^{2}<\frac{4}{K^{2}},
$$

in terms of the normalized wavenumber $K=k v_{0} / \omega_{p}$ and the quantum parameter $H=\hbar \omega_{p} / m_{e} v_{0}^{2}$ (see Fig. 1). ${ }^{7,41}$ We see that when $H=0$, we 


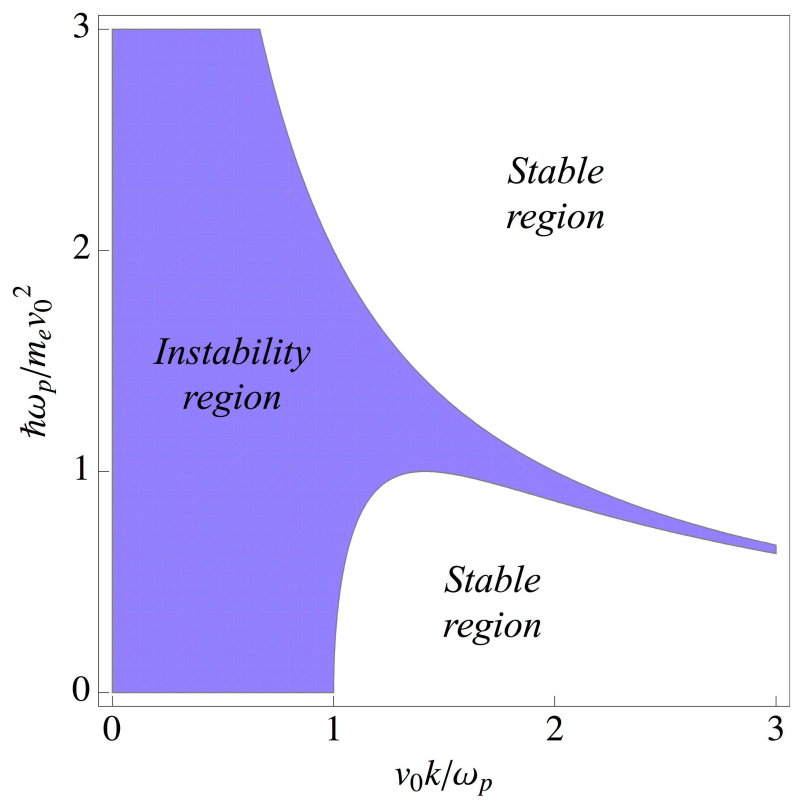

Fig. 1. The regions of stability and instability in the case of the quantum two-stream interaction. ${ }^{7,41}$

have unstable perturbations for $0<K<1$, but when $H \neq 0$ a considerably more complex instability region develops.

A model for treating partial coherence in such systems, based on the Wigner transform technique, ${ }^{42-45}$ can also be developed ${ }^{41}$ (see also Ref. 46). Moreover, using the equations (13) and (14), a similar framework may be set up for electron streams with spin properties.

\subsection{Fluid model}

\subsubsection{Plasmas based on the Schrödinger model}

Suppose that we have $N$ electron wavefunctions, and that the total system wave function can be described by the factorization $\psi\left(\mathbf{x}_{1}, \mathbf{x}_{2}, \ldots \mathbf{x}_{N}\right)=$ $\psi_{1} \psi_{2} \ldots \psi_{N}$. For each wave function $\psi_{\alpha}$, we have a corresponding probability $\mathcal{P}_{\alpha}$. From this, we first define $\psi_{\alpha}=n_{\alpha} \exp \left(i S_{\alpha} / \hbar\right)$ and follow the steps leading to Eqs. (2) and (3). We now have $N$ such equations the wave functions $\left\{\psi_{\alpha}\right\}$. Defining ${ }^{6}$

$$
n \equiv \sum_{\alpha=1}^{N} \mathcal{P}_{\alpha} n_{\alpha}
$$


and

$$
\mathbf{v} \equiv\left\langle\mathbf{v}_{\alpha}\right\rangle=\sum_{\alpha=1}^{N} \frac{\mathcal{P}_{\alpha} n_{\alpha} \mathbf{v}_{\alpha}}{n},
$$

we can define the deviation from the mean flow according to

$$
\mathbf{w}_{\alpha}=\mathbf{v}_{\alpha}-\mathbf{v} .
$$

Taking the average, as defined by (27), of Eqs. (2) and (3) and using the above variables, we obtain the quantum fluid equation

$$
\frac{\partial n}{\partial t}+\nabla \cdot(n \mathbf{v})=0
$$

and

$$
m_{e} n\left(\frac{\partial}{\partial t}+\mathbf{v} \cdot \nabla\right) \mathbf{v}=e n \nabla \phi-\nabla p+\frac{\hbar^{2} n}{2 m_{e}} \nabla\left\langle\left(\frac{\nabla^{2} \sqrt{n_{\alpha}}}{\sqrt{n_{\alpha}}}\right)\right\rangle,
$$

where we have assumed that the average produces an isotropic pressure $p=m_{e} n\left\langle\left|\mathbf{w}_{\alpha}\right|^{2}\right\rangle$ We note that the above equations still contain an explicit sum over the electron wave functions. For typical scale lengths larger than the Fermi wavelength $\lambda_{F}$, we may approximate the last term by the Bohmde Broglie potential ${ }^{6}$

$$
\left\langle\frac{\nabla^{2} \sqrt{n_{\alpha}}}{\sqrt{n_{\alpha}}}\right\rangle \approx \frac{\nabla^{2} \sqrt{n}}{\sqrt{n}}
$$

Using a classical or quantum model for the pressure term, we finally have a quantum fluid system of equations. For a self-consistent potential $\phi$ we furthermore have

$$
\nabla^{2} \phi=\frac{e}{\epsilon_{0}}\left(n-n_{i}\right)
$$

\subsubsection{Spin plasmas}

The collective dynamics of electrons with spin and some of the spin modifications of the classical dispersion relation was presented in Ref. 19. Here we will follow Refs. 19 and 20 for the derivation of the governing equations. Suppose that we have $N$ wave functions for the electrons with magnetic moment $\mu_{e}=-\mu_{B}$, and that, as in the case of the Schrödinger description, the total system wave function can be described by the factorization $\psi=\psi_{1} \psi_{2} \ldots \psi_{N}$. Then the density is defined as in Eq. (26) and the average fluid velocity defined by (27). However, we now have one further fluid variable, the spin vector, and accordingly we let $\mathbf{S}=\left\langle\mathbf{s}_{\alpha}\right\rangle$. From this we 
can define the microscopic microscopic spin density $\mathcal{S}_{\alpha}=\mathbf{s}_{\alpha}-\mathbf{S}$, such that $\left\langle\mathcal{S}_{\alpha}\right\rangle=0$.

Taking the ensemble average of Eqs. (13) we obtain the continuity equation (29), while we the the ensemble average applied to (14) yield

$$
m_{e} n\left(\frac{\partial}{\partial t}+\mathbf{v} \cdot \nabla\right) \mathbf{v}=-e n(\mathbf{E}+\mathbf{v} \times \mathbf{B})-\nabla p+\frac{\hbar^{2} n}{2 m_{e}} \nabla\left(\frac{\nabla^{2} \sqrt{n}}{\sqrt{n}}\right)+\mathbf{F}_{\mathrm{spin}}
$$

and the average of Eq. (19) gives

$$
n\left(\frac{\partial}{\partial t}+\mathbf{v} \cdot \nabla\right) \mathbf{S}=\frac{2 \mu_{B} n}{\hbar} \mathbf{B} \times \mathbf{S}-\nabla \cdot \mathbf{K}+\boldsymbol{\Omega}_{\text {spin }}
$$

respectively. Here the force density due to the electron spin is

$$
\begin{aligned}
\mathbf{F}_{\text {spin }}=- & \frac{2 \mu_{B} n}{\hbar}(\nabla \otimes \mathbf{B}) \cdot \mathbf{S}-\frac{1}{m_{e}} \nabla \cdot[n(\boldsymbol{\Sigma}+\widetilde{\boldsymbol{\Sigma}})] \\
& -\frac{1}{m_{e}} \nabla \cdot\left[n\left(\nabla S_{a}\right) \otimes\left\langle\nabla \mathcal{S}_{\alpha}^{a}\right\rangle+n\left\langle\nabla \mathcal{S}_{\alpha}^{a}\right\rangle \otimes\left(\nabla S_{a}\right)\right],
\end{aligned}
$$

consistent with the results in Ref. 39, while the asymmetric thermal-spin coupling is

$$
\mathbf{K}=n\left\langle\mathbf{w}_{\alpha} \otimes \mathcal{S}_{\alpha}\right\rangle
$$

and the nonlinear spin fluid correction is

$$
\begin{aligned}
& \boldsymbol{\Omega}_{\text {spin }}=\frac{1}{m_{e}} \mathbf{S} \times\left[\partial_{a}\left(n \partial^{a} \mathbf{S}\right)\right]+\frac{1}{m_{e}} \mathbf{S} \times\left[\partial_{a}\left(n\left\langle\partial^{a} \mathcal{S}_{\alpha}\right\rangle\right)\right] \\
& +\frac{n}{m_{e}}\left\langle\frac{\mathcal{S}_{\alpha}}{n_{\alpha}} \times\left\{\partial_{a}\left[n_{\alpha} \partial^{a}\left(\mathbf{S}+\mathcal{S}_{\alpha}\right)\right]\right\}\right\rangle
\end{aligned}
$$

where $\boldsymbol{\Sigma}=\left(\nabla S_{a}\right) \otimes\left(\nabla S^{a}\right)$ is the nonlinear spin correction to the classical momentum equation, $\widetilde{\boldsymbol{\Sigma}}=\left\langle\left(\nabla \mathcal{S}_{(\alpha) a}\right) \otimes\left(\nabla \mathcal{S}_{(\alpha)}^{a}\right)\right\rangle$ is a pressure like spin term (which may be decomposed into trace-free part and trace), and $[(\nabla \otimes \mathbf{B}) \cdot \mathbf{S}]^{a}=\left(\partial^{a} B_{b}\right) S^{b}$. Here the indices $a, b, \ldots=1,2,3$ denotes the Cartesian components of the corresponding tensor. We note that, apart from the additional spin density evolution equation (34), the momentum conservation equation (33) is considerably more complicated compared to the Schrödinger case represented by (30). Moreover, Eqs. (33) and (34) still contains the explicit sum over the $N$ states, and has to be approximated using insights from quantum kinetic theory or some effective theory.

The coupling between the quantum plasma species is mediated by the electromagnetic field. By definition, we let $\mathbf{H}=\mathbf{B} / \mu_{0}-\mathbf{M}$ where $\mathbf{M}=$ 
$-2 n \mu_{B} \mathbf{S} / \hbar$ is the magnetization due to the spin sources. Ampère's law $\nabla \times \mathbf{H}=\mathbf{j}+\epsilon_{0} \partial_{t} \mathbf{E}$ takes the form

$$
\nabla \times \mathbf{B}=\mu_{0}(\mathbf{j}+\nabla \times \mathbf{M})+\frac{1}{c^{2}} \frac{\partial \mathbf{E}}{\partial t},
$$

where $\mathbf{j}$ is the free current contribution The system is closed by Faraday's law

$$
\nabla \times \mathbf{E}=-\frac{\partial \mathbf{B}}{\partial t}
$$

\section{The magnetohydrodynamic limit}

The concept of a magnetoplasma was first introduced in the pioneering work 47 by Alfvén, who showed the existence of waves in magnetized plasmas. Since then, magnetohydrodynamics (MHD) has found applications in a vast range of fields, from solar physics and astrophysical dynamos, to fusion plasmas and dusty laboratory plasmas.

Magnetic fields, an essential component in the MHD description of plasmas, also couples directly to the spin of the electron. Thus, the presence of spin alters the single electron dynamics, introducing a correction to the Lorentz force term. Indeed, from the experimental perspective, a certain interest has been directed towards the relation of spin properties to the classical theory of motion (see, e.g., Refs. 48-60). In particular, the effects of strong fields on single particles with spin has attracted experimental interest in the laser community. ${ }^{50-55}$ However, the main objective of these studies was single particle dynamics, relevant for dilute laboratory systems, whereas our focus will be on collective effects.

We will now include if the ion species, which are assumed to be described by the classical equations and have charge $Z e$, we may derive a set of onefluid equations. ${ }^{20}$ The ion equations read

$$
\frac{\partial n_{i}}{\partial t}+\nabla \cdot\left(n_{i} \mathbf{v}_{i}\right)=0
$$

and

$$
m_{i} n_{i}\left(\frac{\partial}{\partial t}+\mathbf{v}_{i} \cdot \nabla\right) \mathbf{v}_{i}=Z e n_{i}\left(\mathbf{E}+\mathbf{v}_{i} \times \mathbf{B}\right)--\nabla p_{i} .
$$

Next we define the total mass density $\rho \equiv\left(m_{e} n+m_{i} n_{i}\right)$, the centre-ofmass fluid flow velocity $\mathbf{V} \equiv\left(m_{e} n \mathbf{v}_{e}+m_{i} n_{i} \mathbf{v}_{i}\right) / \rho$, and the current density $\mathbf{j}=-e n \mathbf{v}_{e}+Z e n_{i} \mathbf{v}_{i}$. Using these denfinitions, we immediately obtain

$$
\frac{\partial \rho}{\partial t}+\nabla \cdot(\rho \mathbf{V})=0
$$


from Eqs. (29) and (40). Assuming quasi-neutrality, i.e. $n \approx Z n_{i}$, the momentum conservation equations (33) and (41) give

$$
\rho\left(\frac{\partial}{\partial t}+\mathbf{V} \cdot \nabla\right) \mathbf{V}=\mathbf{j} \times \mathbf{B}-\nabla \cdot \boldsymbol{\Pi}-\nabla p+\frac{Z \hbar^{2} \rho}{2 m_{e} m_{i}} \nabla\left(\frac{\nabla^{2} \sqrt{\rho}}{\sqrt{\rho}}\right)+\mathbf{F}_{\text {spin }},
$$

where $\Pi$ is the tracefree pressure tensor in the centre-of-mass frame, and $P$ is the scalar pressure in the centre-of-mass frame. We also note that due to quasi-neutrality, we have $n_{e} \approx Z \rho / m_{i}$ and $\mathbf{v}=\mathbf{V}-m_{i} \mathbf{j} / Z e \rho$, and we can thus express the quantum terms in terms of the total mass density $\rho$, the centre-of-mass fluid velocity $\mathbf{V}$, and the current $\mathbf{j}$. With this, the spin transport equation $(34)$ reads

$$
\rho\left(\frac{\partial}{\partial t}+\mathbf{V} \cdot \nabla\right) \mathbf{S}=\frac{m_{e}}{Z e} \mathbf{j} \cdot \nabla \mathbf{S}+\frac{2 \mu_{B} \rho}{\hbar} \mathbf{B} \times \mathbf{S}-\frac{m_{i}}{Z} \nabla \cdot \mathbf{K}+\frac{m_{i}}{Z} \boldsymbol{\Omega}_{\text {spin }} .
$$

In the momentum equation (43), neglecting the pressure and the Bohmde Broglie potential for the sake of clarity, we have the force density $\mathbf{j} \times$ $\mathbf{B}+\mathbf{F}_{\text {spin }}$. In general, for a magnetized medium with magnetization density $\mathbf{M}$, Ampère's law gives the free current in a finite volume $V$ according to

$$
\mathbf{j}=\frac{1}{\mu_{0}} \nabla \times \mathbf{B}-\nabla \times \mathbf{M}
$$

where we have neglected the displacement current. The surface current is an important part of the total current when we are interested in the forces on a finite volume, as was demonstrated in Ref. 20 and will be shown below.

It it worth noting that the expression of the force density in the momentum conservation equation can, to lowest order in the spin, be derived on general macroscopic grounds. Formally, the total force density on a volume element $V$ is defined as $\mathbf{F}=\lim _{V \rightarrow 0}\left(\sum_{\alpha} \mathbf{f}_{\alpha} / V\right)$, where $\mathbf{f}_{\alpha}$ are the different forces acting on the volume element, and might include surface forces as well. For magnetized matter, the total force on an element of volume $V$ is then

$$
\mathbf{f}_{\text {tot }}=\int_{V} \mathbf{j}_{\text {tot }} \times \mathbf{B} \mathrm{d} V+\oint_{\partial V}(\mathbf{M} \times \hat{\mathbf{n}}) \times \mathbf{B} \mathrm{d} S
$$

where (neglecting the displacement current) $\mathbf{j}_{\text {tot }}=\mathbf{j}+\nabla \times \mathbf{M}$. Inserting the expression for the total current into the volume integral and using the divergence theorem on the surface integral, we obtain the force density

$$
\mathbf{F}_{\text {tot }}=\mathbf{j} \times \mathbf{B}+M_{k} \nabla B^{k},
$$

identical to the lowest order description from the Pauli equation (see Eq. (43)). Inserting the free current expression (45), due to Ampère's law, we 
can write the total force density according to

$$
F^{i}=-\partial^{i}\left(\frac{B^{2}}{2 \mu_{0}}-\mathbf{M} \cdot \mathbf{B}\right)+\partial_{k}\left(H^{i} B^{k}\right) .
$$

The first gradient term in Eq. (48) can be interpreted as the force due to a potential (the energy of the magnetic field and the magnetization vector in that field), while the second divergence term is the anisotropic magnetic pressure effect. Noting that the spatial part of the stress tensor takes the form $^{39}$

$$
T^{i k}=-H^{i} B^{k}+\left(B^{2} / 2 \mu_{0}-\mathbf{M} \cdot \mathbf{B}\right) \delta^{i k},
$$

we see that the total force density on the magnetized fluid element can be written $F^{i}=-\partial_{k} T^{i k}$, as expected. Thus, the Pauli theory results in the same type of conservation laws as the macroscopic theory. The momentum conservation equation (43) then reads

$$
\rho\left(\frac{\partial}{\partial t}+\mathbf{V} \cdot \nabla\right) \mathbf{V}=-\nabla\left(\frac{B^{2}}{2 \mu_{0}}-\mathbf{M} \cdot \mathbf{B}\right)+B^{k} \partial_{k} \mathbf{H}-\nabla p,
$$

where for the sake of clarity we have assumed an isotropic pressure, dropped the displacement current term in accordance with the nonrelativistic assumption, and neglected the Bohm potential (these terms can of course simply be added to (50)). This concludes the discussion of the spin-MHD plasma case. Next, we will look at some applications of the derived equations. However, it should be noted that in many cases the spins are close to thermodynamic equilibrium, and we can thus write the paramagnetic electron response in terms of the magnetization ${ }^{20}$

$$
\mathbf{M}=\frac{\mu_{B} \rho}{m_{i}} \tanh \left(\frac{\mu_{B} B}{k_{B} T}\right) \widehat{\mathbf{B}},
$$

instead of using the full spin dynamics. Here $B$ denotes the magnitude of the magnetic field and $\widehat{\mathbf{B}}$ is a unit vector in the direction of the magnetic field, $k_{B}$ is Boltzmann's constant, and $T$ is the electron temperature.

\section{Examples and applications}

The above equations are quite complicated, but as such also extremely rich. Suitable and physically relevant approximations, such as the magnetization given by the expression (51), will however lead to considerable simplifications. Below we consider two specific examples where such simplifying assumptions lead to interesting spin effects. 


\subsection{Spin solitons}

In Ref. 21, it was shown that the electron spin can introduce novel nonlinear structures in plasmas, with no limiting classical counterpart. In particular, the MHD limit for a electron-positron pair plasma is considered.

Neglecting dissipative effect, the governing equations for the system of interest read

$$
\begin{gathered}
\frac{\partial \rho}{\partial t}+\nabla \cdot(\rho \mathbf{v})=0 \\
\rho\left(\frac{\partial}{\partial t}+\mathbf{V} \cdot \nabla\right) \mathbf{V}=\mathbf{j} \times \mathbf{B}-\nabla \cdot \boldsymbol{\Pi}+\frac{\hbar^{2} \rho}{2 m_{e}^{2}} \nabla\left(\frac{\nabla^{2} \sqrt{\rho}}{\sqrt{\rho}}\right)+\mathbf{F}_{\text {spin }},
\end{gathered}
$$

where $\boldsymbol{\Pi}=\left[\left(T_{e}+T_{p}\right) / 2 m_{e}\right] \mathbf{I}+\left(m^{2} / \rho\right) \mathbf{j} \otimes \mathbf{j}$ is the total pressure tensor in the centre-of-mass frame and

$$
\mathbf{F}_{\mathrm{spin}}=2 \tanh \left(\frac{\mu_{B} B}{k_{B} T}\right) \frac{\mu_{B}}{m_{e}} \nabla B
$$

where we have assumed equal temperature $T$ of the electrons and positrons. Moreover, we have

$$
\frac{\partial \mathbf{B}}{\partial t}=\nabla \times(\mathbf{V} \times \mathbf{B})
$$

while the current is given by

$$
\mathbf{j}=\frac{1}{\mu_{0}} \nabla \times \mathbf{B}-\epsilon_{0} \frac{\partial}{\partial t}(\mathbf{V} \times \mathbf{B})-\frac{\mu_{B}}{2 m} \nabla \times\left[\rho \tanh \left(\frac{\mu_{B} B}{k_{B} T}\right) \hat{\mathbf{B}}\right]
$$

For one-dimensional Alfvén waves, the above system can be reduces to the modified Korteweg-de Vries equation ${ }^{21}$

$$
\left[\frac{\partial}{\partial t}+c_{A, \mathrm{sp}} \cos \theta \frac{\partial}{\partial \xi}+\frac{c_{A}^{3} \cos ^{3} \theta}{2 \omega_{c}^{2} \sin ^{2} \theta} \frac{\partial^{3}}{\partial \xi^{3}}\right] V_{y}=-\frac{\left(\mu_{B} B_{0}\right)^{2}}{2 m_{e} k_{B} T} \frac{V_{y}^{2}}{c_{A}^{3}} \frac{\partial V_{y}}{\partial \xi}
$$

where $c_{A}=\left[c^{2} B_{0}^{2} /\left(c^{2} \mu_{0} \rho_{0}+B_{0}^{2}\right)\right]^{1 / 2}$ is the Alfvén speed, $c_{A, \mathrm{sp}}=c_{A}^{2} /(1+$ $\left.\delta_{\mathrm{sp}}\right)$ is the spin-modified Alfvén speed, $\omega_{c}=e B_{0} / m_{e}$ is the cyclotron frequency, $B_{0}$ is the magnitude of the unperturbed magnetic field, $\rho_{0}$ is the unperturbed density, and we have the spatial coordinate $\xi=x \sin \theta+z \cos \theta$. We see that neglecting the spin contribution leads to a purely dispersive equations. Thus, the spin enables the formation of solitons with no limiting classical solution. 


\subsection{Ferromagnetic plasma behaviour}

For an ion-electron plasma, we have the governing equations ${ }^{25}$

$$
\frac{\partial \rho}{\partial t}+\nabla \cdot(\rho \boldsymbol{V})=0
$$

the momentum equation

$$
\begin{gathered}
\rho\left(\frac{\partial}{\partial t}+\boldsymbol{V} \cdot \boldsymbol{\nabla}\right) \boldsymbol{V}=-\boldsymbol{\nabla}\left(\frac{B^{2}}{2 \mu_{0}}-\boldsymbol{M} \cdot \boldsymbol{B}\right)-\boldsymbol{\nabla} p \\
\quad+\boldsymbol{B} \cdot \boldsymbol{\nabla}\left(\frac{1}{\mu_{0}} \boldsymbol{B}-\boldsymbol{M}\right)+\frac{\hbar^{2} \rho}{2 m_{e} m_{i}} \boldsymbol{\nabla}\left(\frac{\nabla^{2} \sqrt{\rho}}{\sqrt{\rho}}\right),
\end{gathered}
$$

and the idealized Ohm's law

$$
\frac{\partial \boldsymbol{B}}{\partial t}=\boldsymbol{\nabla} \times(\boldsymbol{V} \times \boldsymbol{B}),
$$

where the variables are defined as above. Using the magnetization (51), we obtain a closed set of equations.

In what follows, we will study the linear modes of this system, with a particular focus on the stability properties. With $\rho=\rho_{0}+\rho_{1}, \boldsymbol{B}=\boldsymbol{B}_{0}+\boldsymbol{B}_{1}$, $\boldsymbol{M}=\boldsymbol{M}_{0}+\boldsymbol{M}_{1}$, and $\boldsymbol{v}=\boldsymbol{v}_{1}$, such that $\rho_{1} \ll \rho_{0},\left|\boldsymbol{B}_{1}\right| \ll\left|\boldsymbol{B}_{0}\right|$, $\left|\boldsymbol{M}_{1}\right| \ll\left|\boldsymbol{M}_{0}\right|$, and $\boldsymbol{B}_{0}=B_{0} \hat{\boldsymbol{z}}$, we linearize our equations in the perturbed variables. Assuming that the background quantities are constants, the general dispersion relation can, after a Fourier decomposition, be written

$$
\left(\omega^{2}-k_{z}^{2} \widetilde{C}_{A}^{2}\right)\left[\left(\omega^{2}-k^{2} \widetilde{C}_{A}^{2}-k_{x}^{2} \widetilde{V}_{A}^{2}(k)\right)\left(\omega^{2}-k_{z}^{2} V_{A}^{2}(k)\right)+k_{x}^{2} k_{z}^{2} \widetilde{V}_{A}^{4}(k)\right]=0
$$

where $\widetilde{C}_{A}$ is the spin-modified Alfvén velocity given by

$$
\widetilde{C}_{A}=\frac{C_{A}}{\left[1+\left(\hbar \omega_{p e}^{2} / 2 m_{e} c^{2} \omega_{c e}^{(0)}\right) \tanh \left(\mu_{B} B_{0} / k_{B} T\right)\right]^{1 / 2}},
$$

$C_{A}$ is the standard Alfvén velocity $C_{A}=\left(B_{0}^{2} / \mu_{0} \rho_{0}\right)^{1 / 2}$,

$$
\widetilde{V}_{A}^{2}(k)=V_{A}^{2}(k)-\frac{\hbar \omega_{c e}}{m_{i}} \tanh \left(\frac{\mu_{B} B_{0}}{k_{B} T}\right),
$$

and

$$
V_{A}^{2}(k)=c_{s}^{2}+\frac{\hbar^{2} k^{2}}{4 m_{i} m_{e}}
$$

Here $\omega_{p e}=\left(\rho_{0} e^{2} / \varepsilon_{0} m_{e} m_{i}\right)^{1 / 2}$ is the plasma frequency, $\omega_{c e}^{(0)}$ is the electron cyclotron frequency associated with the external magnetic field (i.e. with the contribution to $B_{0}$ from the spin sources excluded). The relation between 
the full electron cyclotron frequency $\omega_{c e}=e B_{0} / m_{e}$ and $\omega_{c e}^{(0)}$ is given by $\omega_{c e}=\omega_{c e}^{(0)}+\left(\hbar \omega_{p e}^{2} / m_{e} c^{2}\right) \tanh \left(\mu_{B} B_{0} / k_{B} T\right)$. We stress that $\widetilde{V}_{A}$, which to some extent can be considered as an effective acoustic velocity, may be imaginary for a strongly magnetized plasma due to the spin contribution, a fact which will be explored in some detail below.

We consider propagation perpendicular to the external magnetic field, which is the geometry which leads to instability most easily. For the case $\boldsymbol{k}=k \hat{\boldsymbol{x}}$, Eq. (61) reduces to

$$
\begin{gathered}
\omega=k\left[\frac{C_{A}^{2}}{1+\left(\hbar \omega_{p e}^{2} / 2 m_{e} c^{2} \omega_{c e}^{(0)}\right) \tanh \left(\mu_{B} B_{0} / k_{B} T\right)}\right. \\
\left.\quad+c_{s}^{2}+\frac{\hbar^{2} k^{2}}{4 m_{i} m_{e}}-\frac{\hbar \omega_{c e}}{m_{i}} \tanh \left(\frac{\mu_{B} B_{0}}{k_{B} T}\right)\right]^{1 / 2} .
\end{gathered}
$$

The necessary and sufficient instability condition can thus be written as

$$
P_{\mathrm{sp}}+P_{\mathrm{m}}+P+\frac{\rho_{0} \hbar^{2} k^{2}}{4 m_{e} m_{i}}<0,
$$

where the total pressure $P_{\mathrm{tot}}=P_{\mathrm{sp}}+P_{\mathrm{m}}+P$ consists of the effective spin pressure $P_{\mathrm{sp}}=-\left(\rho_{0} \hbar \omega_{c e} / m_{i}\right) \tanh \left(\mu_{B} B_{0} / k_{B} T\right)$, which is the only negative pressure term and therefore the source of the instability, the magnetic pressure $P_{\mathrm{m}}=\rho_{0} C_{A}^{2} /\left[1+\left(\hbar \omega_{p e}^{2} / 2 m_{e} c^{2} \omega_{c e}^{(0)}\right) \tanh \left(\mu_{B} B_{0} / k_{B} T\right)\right]$, and the particle pressure $P=n_{0} m_{i} c_{s}^{2}$, containing both the thermal and Fermi pressure part. Thus, a plasma can contain a magnetization instability, much like the gravitationally induced Jeans instability. ${ }^{25}$

\section{Conclusions and future possibilities}

We have seen that quantum effects, and in particular the electron spin, can introduce new and interesting aspects in plasma theory and experiments. It is expected that the rapid development of quantum plasma theory will be fueled by recent experiments (see, e.g, Ref. 31), and that new regimes of interest will enter the arena as the next-generation laser systems gets online.

Some particular developments that would be of interest is to further look at spin effects from a kinetic perspective. ${ }^{61,62}$ Such treatments would be similar to the density matrix approach, and could, by using analogies from classical kinetic theory, spur the experimental interest in collective quantum effects and the transition from quantum to classical behaviour. Moreover, the fluid equations presented here has not been analyzed to their 
full extent. For example, there are terms which have been neglected, that could produce interesting nonlinear effects in quantum plasmas, such as spin self-interaction. Such topics will be approached in future research.

\section{References}

1. D. Pines, J. Nucl. Energy C: Plasma Phys. 2, 5 (1961).

2. D. Pines, Elementary Excitations in Solids (Westview Press, 1999)

3. D. Kremp, M. Schlanges, and W.-D. Kraeft, Quantum Statistics of Nonideal Plasmas (Springer, 2005).

4. B. Bezzerides and D. F. DuBois, Ann. Phys. (N.Y.) 70, 10 (1972).

5. R. Hakim and J. Heyvaerts, Phys. Rev. A 18, 1250 (1978).

6. G. Manfredi, Fields Inst. Comm 46, 263 (2005)

7. F. Haas, G. Manfredi, and M. R. Feix, Phys. Rev. E 62, 2763 (2000).

8. F. Haas, Phys. Plasmas 12, 062117 (2005).

9. L. G. Garcia, F. Haas, L. P. L. de Oliveira, and J. Goedert, Phys. Plasmas 12, $012302(2005)$

10. P. K. Shukla, Phys. Lett. A 352, 242 (2006).

11. F. Haas, L. G. Garcia, J. Goedert, and G. Manfredi, Phys. Plasmas 10, 3858 (2003).

12. L. G. Garcia, F. Haas, L. P. L. de Oliviera, and J. Goedert, Phys. Plasmas 12, $012302(2005)$

13. P. K. Shukla and L. Stenflo, Phys. Lett. A 355, 378 (2006).

14. P. K. Shukla, Phys. Lett. A 357, 229 (2006).

15. P. K. Shukla, L. Stenflo, and R. Bingham, Phys. Lett. A 359, 218 (2006).

16. P. K. Shukla and B. Eliasson, Phys. Rev. Lett. 96, 245001 (2006).

17. P. K. Shukla, S. Ali, L. Stenflo, and M. Marklund, Phys. Plasmas 13, 112111 (2006).

18. F. Haas, Europhys. Lett. 44, 45004 (2007).

19. M. Marklund and G. Brodin, Phys. Rev. Lett. 98, 025001 (2007).

20. G. Brodin and M. Marklund, New J. Phys. 9, 277 (2007).

21. G. Brodin and M. Marklund, Phys. Plasmas 14, 112107 (2007).

22. P. K. Shukla and B. Eliasson, Phys. Rev. Lett. 96, 245001 (2006).

23. P. K. Shukla and B. Eliasson, Phys. Rev. Lett. 99, 096401 (2007).

24. D. Shaikh and P. K. Shukla, Phys. Rev. Lett. 99, 125002 (2007).

25. G. Brodin and M. Marklund, Phys. Rev. E, in press (2007). (arXiv:0709.3575)

26. H. G. Craighead, Science 290, 1532 (2000).

27. G. Manfredi and P.-A. Hervieux, Appl. Phys. Lett. 91, 061108 (2007).

28. W. Li, P. J. Tanner, and T. F. Gallagher, Phys. Rev. Lett. 94, 173001 (2005).

29. R. S. Fletcher, X. L. Zhang, and S. L. Rolston, Phys. Rev. Lett. 96, 105003 (2006).

30. A. K. Harding and D. Lai, Rep. Prog. Phys. 69, 2631 (2006).

31. S. H. Glenzer et al., Phys. Rev. Lett. 98, 065002 (2007).

32. M. Marklund and P. K. Shukla, Rev. Mod. Phys. 78, 591 (2006).

33. E. Lundström et al., Phys. Rev. Lett. 96, 083602 (2006). 
34. G. Brodin, M. Marklund, B. Eliasson and P. K. Shukla, Phys. Rev. Lett. 98, $125001(2007)$

35. J. Lundin, J. Zamanian, M. Marklund and G. Brodin, Phys. Plasmas, 14, 062112 (2007).

36. P. R. Holland, The Quantum Theory of Motion (Cambridge University Press, Cambridge, 1993).

37. P. A. M. Dirac, Principles of Quantum Mechanics (Oxford University Press, Oxford, 1981).

38. A. O. Barut and W. D. Thacker, Phys. Rev. D 31, 2076 (1985).

39. S. R. de Groot and L. G Suttorp, Foundations of Electrodyanmics (NorthHolland, 1972).

40. J. Dawson, Phys. Fluids 4, 869 (1961).

41. D. Anderson, B. Hall, M. Lisak, and M. Marklund, Phys. Rev. E 65, 046417 (2002).

42. E. P. Wigner, Phys. Rev. 40, 749 (1932).

43. J. E. Moyal, Proc. Cambridge Philos. Soc. 45, 99 (1949).

44. J. T. Mendonça, Theory of Photon Acceleration (IOP Publishing, 2001).

45. W. P. Schleich, Quantum Optics in Phase Space (Wiley, 2001).

46. M. Marklund, Phys. Plasmas 12, 082110 (2005).

47. H. Alfvén, Nature 150, 405 (1942).

48. B. I. Halperin and P. C. Hohenberg, Phys. Rev. 188, 898 (1969).

49. A. V. Balatsky, Phys. Rev. B 42, 8103 (1990).

50. U. W. Rathe, C. H. Keitel, M. Protopapas, and P. L. Knight, J. Phys. B: At. Mol. Opt. Phys. 30, L531 (1997).

51. S. X. Hu and C. H. Keitel, Phys. Rev. Lett. 83, 4709 (1999).

52. R. Arvieu, P. Rozmej, and M. Turek, Phys. Rev. A 62, 022514 (2000).

53. J. R. Vázquez de Aldana and L. Roso, J. Phys. B: At. Mol. Opt. Phys. 33, 3701 (2000).

54. M. W. Walser and C. H. Keitel, J. Phys. B: At. Mol. Opt. Phys. 33, L221 (2000).

55. M. W. Walser, D. J. Urbach, K. Z. Hatsagortsyan, S. X. Hu, and C. H. Keitel, Phys. Rev. A 65, 043410 (2002).

56. Z. Qian and G. Vignale, Phys. Rev. Lett. 88, 056404 (2002).

57. J. S. Roman, L. Roso, and L. Plaja, J. Phys. B: At. Mol. Opt. Phys. 37, 435 (2004).

58. R. L. Liboff, Europhys. Lett. 68, 577 (2004).

59. J. N. Fuchs, D. M. Gangardt, T. Keilman, and G. V. Shlyapnikov, Phys. Rev. Lett. 95, 150402 (2005).

60. K. Kirsebom et al., Phys. Rev. Lett. 87, 054801 (2001).

61. S. C. Cowley, R. M. Kulsrud, and E. Valeo, Phys. Fluids 29, 430 (1986).

62. R. M. Kulsrud, E. J. Valeo, and S. C. Cowley, Nucl. Fusion 26, 1443 (1986). 